\title{
¿Meningioma en placa o meningioma intraóseo?
}

\section{En Plaque Meningioma or Intraosseous Meningioma?}

\author{
Diego Páez-Granda ${ }^{1}$ Francisca Velázquez-Marín ${ }^{1}$ \\ ${ }^{1}$ Hospital Universitario Virgen de la Arrixaca, Murcia, España \\ Rev Argent Radiol 2018;82:134-136.
}

Address for correspondence Diego Páez Granda, Pedro García Villalba número 28, piso 3 puerta A, La Alberca, Murcia, Código postal: 30150, España (e-mail: drdiegopaez@hotmail.com).
Estimados Editores

La valoración de la patología tumoral intracraneal es un motivo muy frecuente de solicitud de pruebas imagenológicas, por lo que conocer las características radiológicas de la mayoría de esas lesiones es imprescindible para el correcto desempeño del radiólogo dentro del equipo médico. Habitualmente nos enfrentamos a neoplasias relativamente comunes que no suponen una mayor dificultad en el diagnóstico. Sin embargo, en algunas ocasiones, observamos presentaciones atípicas de tumores que, por el desconocimiento de sus hallazgos característicos, las confundimos con lesiones de similar aspecto. El objetivo de este trabajo es presentar ante ustedes el caso de una presentación atípica de un tumor intracraneal muy frecuente.

Paciente femenina de 41 años con gestación de 32 semanas y antecedente de hipertiroidismo no tratado, acudió a urgencias por presentar dolor retro-orbitario de predominio derecho y evolución subaguda. La sintomatología era continua, tenía un empeoramiento vespertino y respondía parcialmente a la administración de analgésicos simples. En el examen físico se detectó distopia y proptosis del ojo derecho, acompañadas de ptosis palpebral y midriasis homolaterales. Se observó también limitación parcial para la supraducción, aducción e inclinotorsión del ojo derecho, con diplopía binocular en dichas posiciones. Ante la sospecha de patología en la hendidura esfenoidal se decidió realizar resonancia magnética (RM) cerebral sin contraste. Esa prueba reveló un engrosamiento del ala mayor del esfenoides derecho, la cual mantenía una señal hipointensa homogénea en secuencias ponderadas en T1 y T2 ( - Fig. 1). Ante esos hallazgos, se decidió ampliar el estudio con la administración de contraste intravenoso. En esas secuencias, se observó un intenso realce de la duramadre subyacente al hueso esfenoidal engrosado, acompañado de un tenue aumento de señal en el espesor del hueso engrosado e invasión de las estructuras adyacentes (seno cavernoso y silla turca) (-Fig. 2). Considerando los hallazgos descriptos, se sugirió el diagnóstico de meningioma en placa.

Los meningiomas son los tumores primarios intracraneales no gliales más frecuentes y habitualmente se localizan en el espacio subdural. ${ }^{1}$ Los meningiomas en placa son un subgrupo menos frecuentemente observado de esos tumores, caracterizados por un patrón de crecimiento que asocia una reacción hiperostótica severa del hueso adyacente. ${ }^{2}$ Ese tipo de presentación tumoral ocurre más frecuentemente en mujeres, y se localiza principalmente en las alas esfenoidales o en la convexidad cerebral. ${ }^{2,3}$ La clínica depende de la localización y evolución del crecimiento tumoral, por lo que los pacientes pueden ser asintomáticos, o presentar déficits asociados a las estructuras con las que la lesión contacta, como proptosis, déficits visuales, cefalea o incluso convulsiones. ${ }^{4}$ Los estudios de tomografía computada (TC) sin contraste, son útiles para detectar las alteraciones óseas, pero no permiten distinguir la hiperostosis del componente dural. ${ }^{5}$ El estudio de RM con administración de contraste es de elección para el diagnóstico de esa patología, porque permite delimitar el hueso hiperostótico y el tumor meningeo. ${ }^{5}$ Existe una patología de características radiológicas similares, con la que la diferenciación mediante pruebas de imagen es casi imposible. ${ }^{6,7}$ Esos tumores llamados meningiomas intraóseos son un subtipo de lesiones que representan menos del $1 \%$ de todos los meningiomas y se originan de células aracnoideas residuales localizadas en el interior del hueso (meningioma extradural). ${ }^{7,8}$ Si bien esa es una entidad independiente, Rietzy $y$ col. Demostraron, en una serie de 79 casos de pacientes con meningiomas intratemporales, que más del $70 \%$ de ellos compartían un componente intradural, siendo exclusivamente intraóseos tan solo el $25 \%{ }^{9}$

La detección de un claro realce dentro del hueso tras la administración de contraste y la escasa afectación dural en relación con el grado de hiperostosis son signos que deben received

May 31, 2017

accepted

September 10, 2017

published online

March 26, 2018
DOI https://doi.org/

$10.1055 / \mathrm{s}-0038-1639490$.

ISSN 1852-9992.
Copyright (c) 2019, Sociedad Argentina de Radiología. Publicado por Thieme Revinter Publicações Ltda., Rio de Janeiro, Brazil. Todos los derechos reservados.
License terms

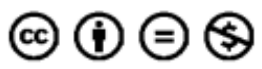




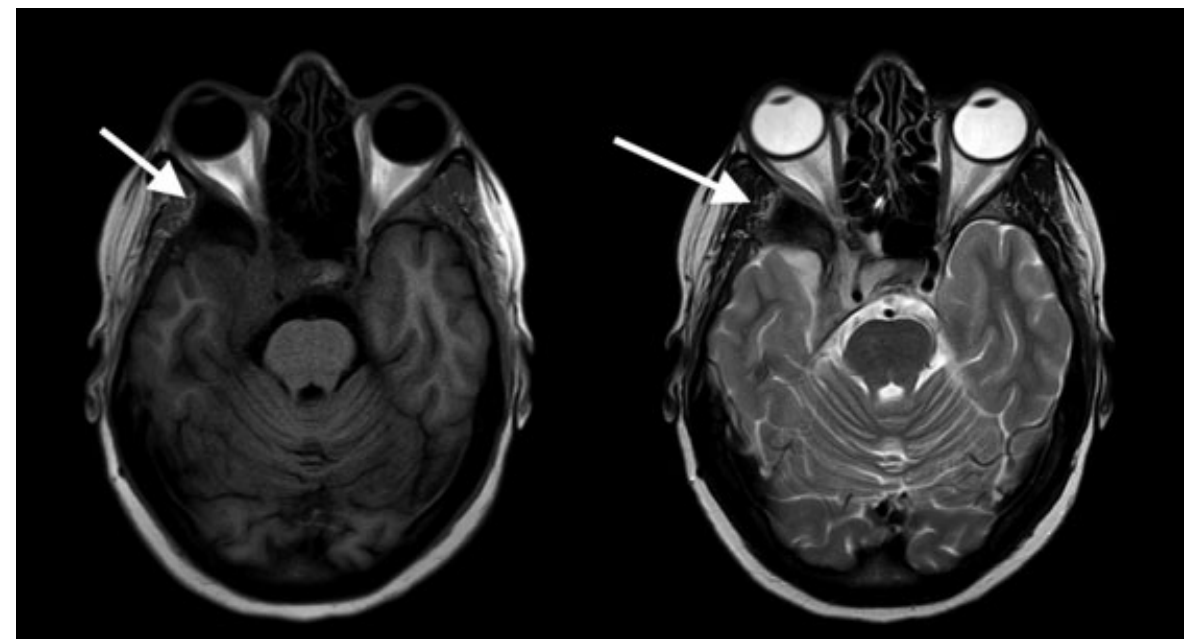

Fig. 1 Resonancia magnética (RM) cerebral, secuencias ponderadas en Ty T2, cortes axiales. Se aprecia un severo engrosamiento del ala mayor del esfenoides derecho, que mantiene una señal hipointensa homogénea (flechas blancas).

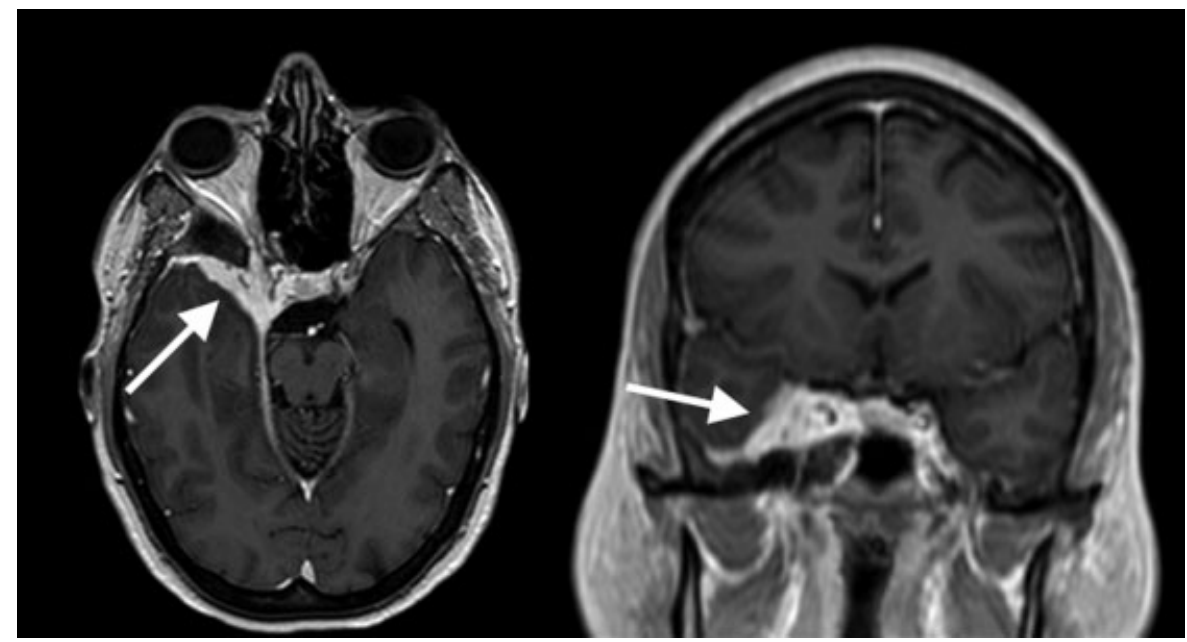

Fig. 2 RM cerebral en secuencias ponderadas en 3DT1 tras la administración de contraste, cortes axial y coronal. Se observa un intenso realce homogéneo de la duramadre engrosada subyacente al esfenoides, sugestivo de meningioma en placa (flechas blancas). Esos hallazgos se acompañan de un tenue y focal aumento de señal en el espesor del hueso engrosado, e invaden las estructuras adyacentes, por lo que se extienden hacia el seno cavernoso y la silla turca.

sugerir al radiólogo la presencia de meningioma intraóseo. ${ }^{6}$ Debemos considerar que esos hallazgos se atribuyen al meninigioma intraóseo osteoblástico, ya que existe una presentación atípica en la que la lesión tiene un comportamiento predominantemente osteolítico, adoptando un aspecto que no es considerado dentro del diagnóstico diferencial de ese tipo de tumor. ${ }^{6}$ Otra entidad de la que se debe diferenciar el meningioma en placa es la displasia fibrosa. ${ }^{8}$ Kim y col., han propuesto que un patrón de hiperostosis periosteal, la irregularidad superficial del hueso hiperostótico y los cambios intracraneales como el edema cerebral, deben sugerir el diagnóstico de meningioma en placa sobre displasia. ${ }^{8}$ Otras lesiones de las que debemos diferenciar esa enfermedad son las metástasis durales y craneales. La elección del tratamiento depende de la evaluación del crecimiento tumoral y de la afectación de estructuras vecinas. ${ }^{10}$ Debemos considerar que esos tumores invaden pequeños canales intraóseos, así como planos subcutáneos, características que generan una tendencia a la recurrencia tumoral. ${ }^{7}$

\section{Confidencialidad de los datos}

Los autores declaran que han seguido los protocolos de su centro de trabajo sobre la publicación de datos de pacientes y que todos los pacientes incluidos en el estudio han recibido información suficiente y han dado su consentimiento informado por escrito para participar en dicho estudio.

\section{Conflicto de intereses}

Los autores declaran no tener ningún conflicto de intereses. 


\section{Bibliografía}

1 Wiemels J, Wrensch M, Claus EB. Epidemiology and etiology of meningioma. J Neurooncol 2010;99(03):307-314

2 Simas NM, Farias JP. Sphenoid Wing en plaque meningiomas: Surgical results and recurrence rates. Surg Neurol Int 2013;4:86

3 Watts J, Box G, Galvin A, Brotchie P, Trost N, Sutherland T. Magnetic resonance imaging of meningiomas: a pictorial review. Insights Imaging 2014;5(01):113-122

4 Zakaria W, Taha A. Hyperostotic sphenoid wing meningioma en plaque: proptosis management. Romanian Neurosurgery. 2016; 4:498-506

5 Buetow MP, Buetow PC, Smirniotopoulos JG. Typical, atypical, and misleading features in meningioma. Radiographics 1991;11(06): 1087-1106
6 Elder JB, Atkinson R, Zee CS, Chen TC. Primary intraosseous meningioma. Neurosurg Focus 2007;23(04):E13

7 Vrionis FD, Robertson JH, Gardner G, Heilman CB. Temporal bone meningiomas. Skull Base Surg 1999;9(02):127-139

8 Saat R, Lempinen L, Laulajainen-Hongisto A, Markkola A, Jero J. An En Plaque Meningioma of the Temporal Bone, Complicating with a Cholesteatoma, Chronic Otitis Media and an Intracranial Abscess. Ann Otolaryngol Rhinol. 2015;2:1050

9 Rietz DR, Ford CN, Kurtycz DF, Brandenburg JH, Hafez GR. Significance of apparent intratympanic meningiomas. Laryngoscope 1983;93 (11 Pt 1):1397-1404

10 Schick U, Bleyen J, Bani A, Hassler W. Management of meningiomas en plaque of the sphenoid wing. J Neurosurg 2006;104(02): 208-214 